\title{
All-optical Multi-level Phase Quantization Based on Phase Sensitive Amplification with Low-order Harmonics
}

\author{
Hongxiang Wang, Member, IEEE, Limeng Pan, Feifei Lu, Ganapathy Senthil Murugan, Yongmei Sun, \\ Yuefeng Ji, Senior Member, IEEE,
}

\begin{abstract}
A novel scheme is proposed for all-optical multilevel phase quantization by mixing two lower-order harmonics, rather than mixing the signal with its conjugate $(M-1) t h$ harmonic which is difficult to be generated but necessary for the traditional quantization method. The low-order harmonics used in the proposed scheme are determined as the conjugate $(M / 2-1) t h$ and the $(M / 2+1) t h$ harmonics for $M=4 n$, or the conjugate $(M / 2-2)$ th and the $(M / 2+2)$ th harmonics for $M=4 n+2$, or the conjugate $[(M-1) / 2]$ th and the $[(M+1) / 2]$ th harmonics for $M=2 n+1, n=1,2,3 \ldots$. The simulations show the effectiveness of the scheme for the eightand nine-level all-optical phase quantization. Furthermore, the application of the scheme to the all-optical phase regeneration is validated. An improved method with two cascading stages is also proposed and validated to achieve a monotonic step-like phase-phase transfer characteristic for the optimized all-optical phase quantization. This proposed scheme provides a new way for multi-level phase quantization and multi-level phase shift keying (MPSK) regeneration to meet the ever increasing demand for the bandwidth in fiber optic communication.
\end{abstract}

Index Terms-All-optical signal processing, phase quantization, phase regeneration, phase sensitive amplification, low-order harmonic, phase shift keying.

\section{INTRODUCTION}

$\mathbf{T}$ HE all-optical signal processing provides benefits over the electric signal processing with higher speed, lower latency and larger-bandwidth provisioning [1], [2]. The all-optical quantization is a crucial part of optical signal processing. There have been already many studies on optical amplitude quantization [3]-[6]. However, optical phase quantization has recently attracted considerable attention in optical signal processing field such as optical analog-to-digital conversion (ADC) and regeneration of phase encoding signals in fiber communications [7]-[11]. In terms of optical ADC, quantization precision is a critical factor to influence the result. The more levels of the signal is quantized, the less quantization error will be. To meet the ever increasing demand

Manuscript received 24 July, 2018; revised XXX XX, XXXX. This work was supported in part by the National Natural Science Foundation of China (NSFC) (61331008). (Corresponding author: Hongxiang Wang.)

H. Wang, L. Pan, F. Lu, Y. Sun and Y. Ji are with the State Key Laboratory of Information Photonics and Optical Communications, School of Information and Communication Engineering, Beijing University of Posts and Telecommunications, Beijing 100876, China ( e-mail: wanghx@ bupt.edu.cn; panlimeng@bupt.edu.cn; luffxing@163.com; ymsun@bupt.edu.cn; jyf@bupt.edu.cn).

Ganapathy Senthil Murugan is with the Optoelectronics Research Centre, University of Southampton, Southampton SO17 1BJ, U.K. (e-mail: smg@orc.soton.ac.uk). for the bandwidth in fiber optic communication [12], [13], the multi-level phase shift keying (MPSK) which provides higher spectral efficiency is of great importance in all-optical networking.

All-optical phase quantization can be realized by using phase sensitive amplification (PSA) [14]-[17]. The traditional all-optical phase quantization is achieved through mixing the original signal and its conjugate $(M-1)$ th phase harmonic generated from the signal using a cascaded four wave mixing (FWM) process in a nonlinear optical medium such as highly nonlinear fiber (HNLF). However, the applicability of traditional method to higher-level quantization is weak due to the difficulties of generating the $(M-1) t h$ phase harmonic when $M$ is large. In addition, the amplitude ratio between the original signal and the conjugate $(M-1) t h$ harmonic makes a great influence on the effect of phase quantization and it is not easy to control the amplitude ratio in the traditional scheme. To improve the applicability of the scheme to higher-level quantization, Jeng-Yuan Yang et al. proposed a new dual-pump degenerate PSA structure, which uses the two $(M / 2-1) t h$ phase harmonic conjugates as the two pumps to generate the conjugate $(M-1)$ th phase harmonic [18]. Although the scheme improves the efficiency of generating the needed harmonic, the two phase harmonic conjugates are generated from two different nonlinear process, and it is difficult to make the symbols of the two harmonics synchronous. More importantly, in this scheme the amplitude ratio between the signal and its corresponding harmonic still can't be adjusted directly. To solve the problem, Takayuki Kurosu et al. proposed a scheme named hybrid optical phase squeezer (HOPS) to perform phase regeneration through the coherent addition of a phase-conjugate copy of the signal and a $(M-1)$ th phase harmonic by a frequency shifter [19]. It separates the process of coherent addition from nonlinear process and realizes the controlling of power ratio between the signal and its harmonic. However, it is difficult to achieve the higher level phase quantization owing to the difficulty of generating the $(M-1)$ th phase harmonic of the signal when $M$ is large. Besides, the signal after regeneration by HOPS is not at the signal frequency.

In this paper, a novel scheme of all-optical phase quantization is proposed which realizes the $M$-level quantization by the interference of two low-order harmonics of order $\sim M / 2$ generated from the same nonlinear process. The proposed method decreases the order number of necessary harmonics and avoids 
the difficulty of high-order harmonics generating. It becomes more effectual and easier for multi-level phase quantization or MPSK regeneration when $M$ is large. This proposed scheme also makes it possible to adjust the amplitude ratio between the two harmonics directly owing to the use of a phase modulator (PM) which plays the role as frequency shifter. The rest of this paper is organized as follows. In section II, the principle of the proposed scheme is described and the eight- and ninelevel quantization are introduced as examples in detail. In section III, we present the simulation configuration of the scheme. In section IV, the simulated results of the eight- and nine-level quantization are given, and the application of the eight-level quantization principle to 8PSK phase regeneration is discussed. The simulation of phase-preserving amplitude regeneration of 8PSK signal is performed following the phase regeneration device. An improved method with two cascading stages is simulated and achieves a monotonic step-like phasephase transfer characteristic for the all-optical 8PSK phase quantization. In section $\mathrm{V}$, the conclusion is drawn.

\section{Theory AND Operating PRINCIPLE}

In traditional scheme, $M$-level phase quantization can be achieved by coherently adding two waves, which are the signal and the conjugate $(M-1)$ th harmonic [1]. The transfer function is given by [1]:

$$
\left|A_{\text {out }}\right| e^{i \varphi_{\text {out }}}=e^{i \varphi_{\text {in }}}+m e^{-i \varphi_{\text {in }}(M-1)}
$$

Here, $A_{\text {out }}$ and $\varphi_{\text {out }}$ are the amplitude and phase of the quantized output signal. $\varphi_{i n}$ is the phase of the input signal. $m$ is the amplitude ratio between the conjugate $(M-1) t h$ harmonic and the input signal. It is difficult to realize the $M$ level quantization when $M$ is large due to the difficulty of generating the conjugate $(M-1) t h$ harmonic. In this study, a new approach is proposed for the all-optical phase quantization by coherently adding two lower-order harmonics. Supposing the two harmonics' orders are $x$ and $y$ respectively, $x$ and $y$ should satisfy the following conditions: $|x|+|y|=M$, by which the $M$-level phase quantization can be achieved; $x$ and $y$ should have no common divisor, otherwise a $M / d$-level quantization will be achieved instead of the $M$-level quantization, where $d$ is the greatest common divisor of $|x|$ and $|y|$; the values of $|x|$ and $|y|$ should be close enough to each other to make $|x|$ and $|y|$ as small as possible and easy to be generated. According to the above conditions, the two lower-order harmonics will be the conjugate $(M / 2-1) t h$ and the $(M / 2+1) t h$ harmonics for $M=4 n$, or the conjugate $(M / 2-2) t h$ and the $(M / 2+2) t h$ harmonics for $M=4 n+2$, or the conjugate $[(M-1) / 2]$ th and the $[(M+1) / 2]$ th harmonics for $M=2 n+1, n=1,2,3 \ldots$. Then, the highest harmonic order needed for the $M$-level alloptical phase quantization will be $(M / 2+1)$ if $M=4 n$, or $(M / 2+2)$ if $M=4 n+2$, or $(M+1) / 2$ if $M=2 n+1$, rather than $(M-1)$. The order of harmonic needed for the $M$-level all-optical phase quantization can be reduced about a half comparing to the traditional approach as shown in Eq. (1)
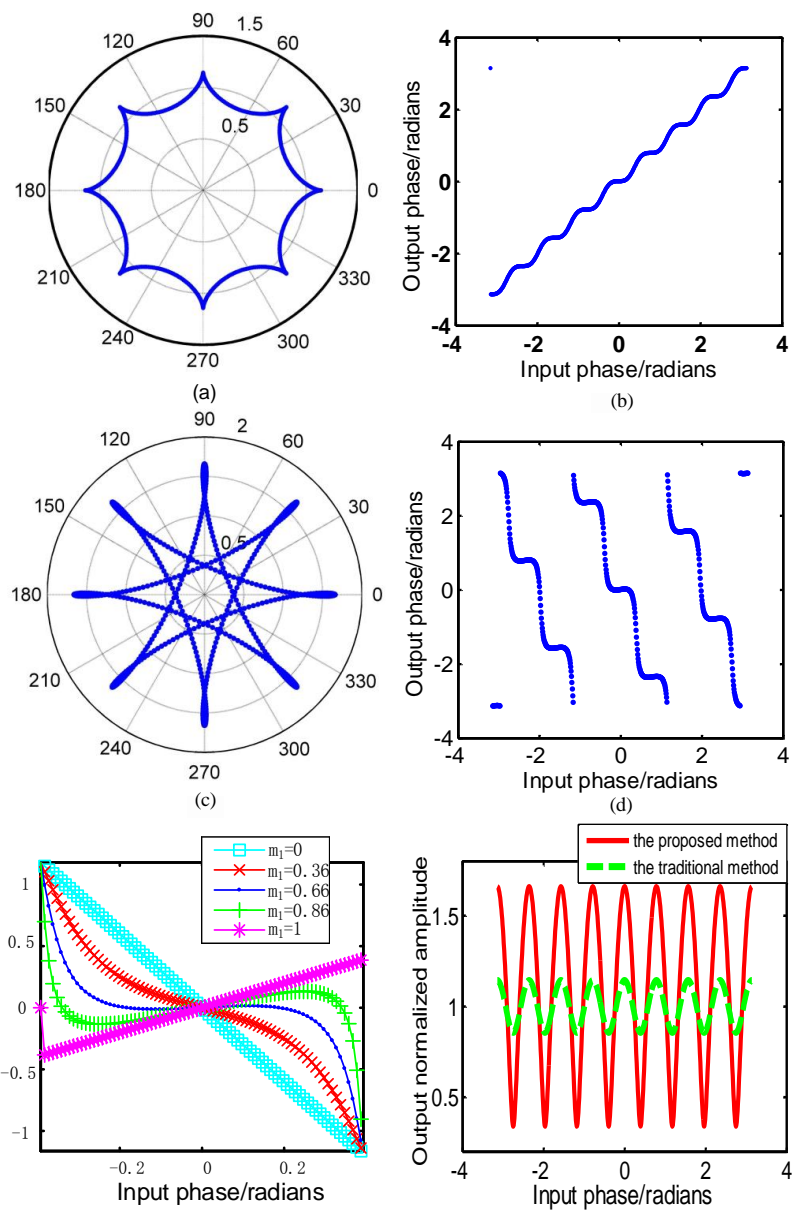

(f)

Fig. 1. (a) Constellation diagram and (b) phase-phase transfer characteristic of the traditional method as Eq. (1) for $m=1 / 7$; (c) constellation diagram, (d) phase-phase transfer characteristic of the proposed method as Eq. (3) for $m_{1}=0.66$, and (e) phase-phase characteristics with different values of amplitude ratio of the proposed method using Eq. (3); (f) the phase-amplitude characteristics of the proposed method and the traditional method for eightlevel phase quantization.

when $M$ is large. The specific equation of achieving the $M$ level all-optical phase quantization can be written as follows:

$$
\begin{aligned}
& \left|A_{\text {out }}\right| e^{i \varphi_{\text {out }}}= \\
& \left\{\begin{array}{l}
e^{-i \varphi_{\text {in }}(M / 2-1)}+m_{1} \mathrm{e}^{i \varphi_{\text {in }}(M / 2+1)}, M=4 n \\
e^{-i \varphi_{\text {in }}(M / 2-2)}+m_{11} e^{i \varphi_{\text {in }}(M / 2+2)}, M=4 n+2 \\
e^{-i \varphi_{\text {in }}(M-1) / 2}+m_{2} \mathrm{e}^{i \varphi_{\text {in }}(M+1) / 2}, M=2 n+1
\end{array}\right.
\end{aligned}
$$

Where, $M>2, n=1,2,3 \ldots$; the amplitude of the $(M / 2-$ 1)th, $(M / 2-2) t h$, and $[(M-1) / 2] t h$ conjugate harmonics are normalized; the $m_{1}, m_{11}$, and $m_{2}$ are the amplitude ratio between the two harmonics that add coherently when $M=4 n$, $M=4 n+2$, and $M=2 n+1$, respectively.

In Eq. (2), the phase differences between the two harmonics adding coherently are all $M \varphi_{\text {in }}$ for the three cases. The two harmonics will interfere constructively and destructively for $M \varphi_{i n}=2 k \pi$ and $M \varphi_{i n}=(2 k+1) \pi, k=1,2,3 \ldots$, respectively. Thus, the $M$-level all-optical phase quantization is achieved. 


\section{A. Eight-level Phase Quantization}

In order to provide a thorough insight of the operation principle when $M$ is even, we take the eight-level phase quantization as an example $(M=8)$. Supposing the input signal is free of amplitude noise, if normalized, it can be described as $\left|A_{i n}\right| e^{i \varphi_{\text {in }}}=e^{i \varphi_{\text {in }}}$ [16]. Its $(M / 2-1=3) r d$ phase harmonic conjugate and $(M / 2+1=5)$ th phase harmonic are necessary for the eight-level all-optical phase quantization according to the Eq. (2), and the output signal can be described as follows:

$$
\left|A_{\text {out }}\right| e^{i \varphi_{\text {out }}}=e^{-i 3 \varphi_{\text {in }}}+m_{1} e^{i 5 \varphi_{\text {in }}}
$$

Where, $m_{1}$ is the amplitude ratio between the 5th phase harmonic and the 3rd phase harmonic conjugate. The constellation diagram and the phase-phase transfer characteristic of the eight-level phase quantization using the traditional method as Eq. (1) are illustrated in Fig. 1(a) and (b) with $\varphi_{i n}$ ranging from $-\pi$ to $\pi$ and optimized $m=1 / 7$, respectively. The constellation diagram and the phase-phase transfer characteristic of the eight-level phase quantization using the proposed method of Eq. (3) are respectively illustrated in Fig. 1(c) and(d), with $\varphi_{\text {in }}$ ranging from $-\pi$ to $\pi$ and optimized $m_{1}=0.66$. When the input phase is within the range of $\{(-9 \pi / 8,-7 \pi / 8),(-7 \pi / 8,-5 \pi / 8)$, $(-5 \pi / 8,-3 \pi / 8),(-3 \pi / 8,-\pi / 8),(-\pi / 8, \pi / 8),(\pi / 8,3 \pi / 8)$, $(3 \pi / 8,5 \pi / 8),(5 \pi / 8,7 \pi / 8)\}$, the corresponding output phase is located at $\{-\pi, \pi / 4,-\pi / 2,3 \pi / 4,0,-3 \pi / 4, \pi / 2,-\pi / 4\}$, respectively. However, for the traditional method, the corresponding output phase is located at $\{-\pi,-3 \pi / 4,-\pi / 2$, $-\pi / 4,0, \pi / 4, \pi / 2,3 \pi / 4\}$, respectively. Although the constellation diagram and the phase-phase transfer characteristic achieved by Eq. (3) are not quite the same as those obtained by using the traditional method as Eq. (1), and the input-tooutput phase response is not monotonic, they are quite ideal because we only focus on quantizing the analog signal to distinct discrete phase levels for the phase quantization. It is clearly seen from Fig. 1(c) and (d) that the phases of $\varphi=0$, $\pm \pi / 4, \pm \pi / 2, \pm 3 \pi / 4$, and $\pi$ are amplified and the quadrature phases are attenuated. Thus, the eight-level all-optical phase quantization is achieved. In addition, the amplitude ratio $m_{1}$ has a great impact on the result of quantization. The phasephase transfer characteristics with different $m_{1}$ are shown in Fig. 1(e), for $\varphi_{i n}$ ranging from $-\pi / 8$ to $\pi / 8$, where $m_{1}=0.66$ delivers clearly visible eight-level all-optical phase quantization effect [20].

The phase-to-amplitude characteristics of the traditional and the proposed method are illustrated in Fig. 1 (f) when amplitude ratio between signals and the corresponding harmonics are all set to be the optimum. It is obvious that the phase-toamplitude response of the proposed method is sharper than that in traditional method. Specifically, the maximum amplitude after quantization is 1.66 and the minimum amplitude is 0.34 when the amplitude of input full-phase signal is 1 and optimized $m_{1}=0.66$. Hence the extinction ratio (EXT) can be calculated as $E X T=10 \log \left(1.66^{2} / 0.34^{2}\right)=13.77 \mathrm{~dB}$. On the phase-phase transfer curve, the phase difference between two adjacent steps is $3 \pi / 4$. However, in traditional scheme, the

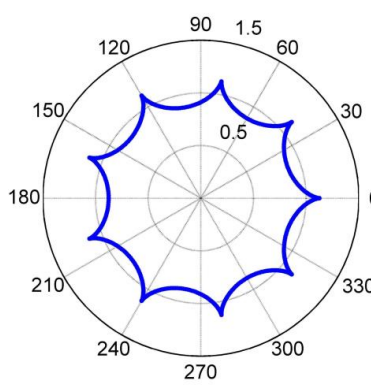

(a)
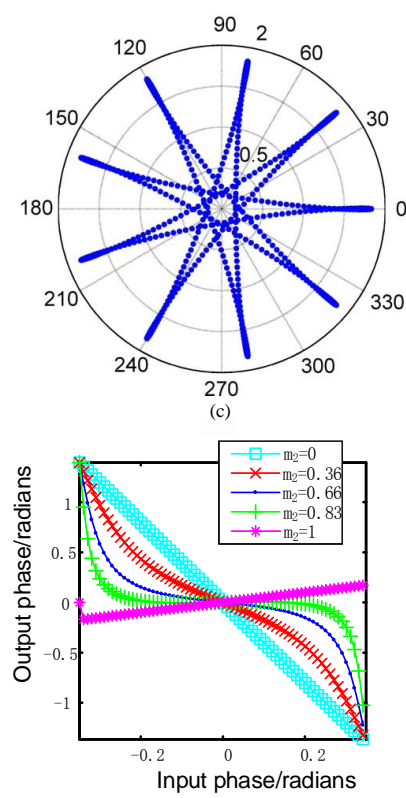

(e)

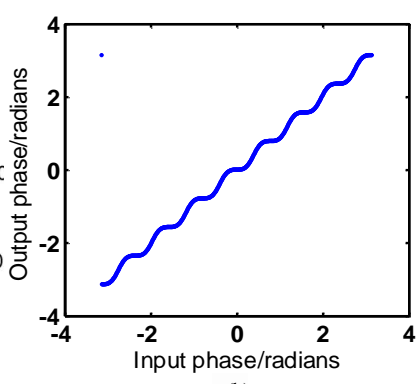

(b)
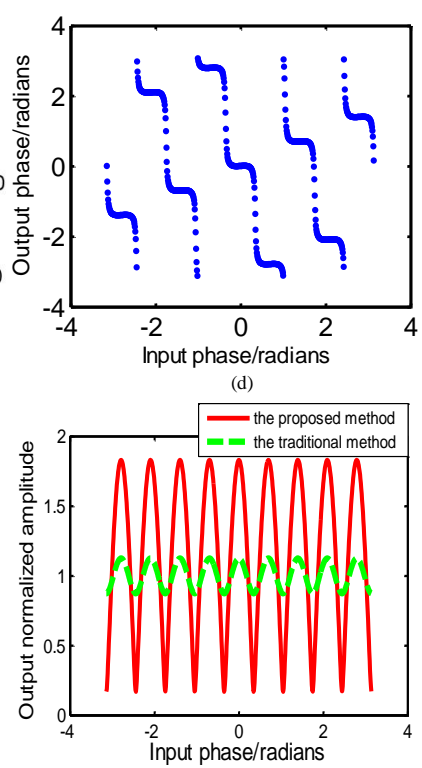

(f)
Fig. 2. (a) Constellation diagram and (b) phase-phase transfer characteristic of the traditional method as Eq. (1) for $m=1 / 8$; (c) constellation diagram, (d) phase-phase transfer characteristic of the traditional method as Eq. (4) for $m_{2}=0.83$, and (e) phase-phase characteristics with different values of amplitude ratio of the proposed method using Eq. (4); (f) the phase-amplitude characteristics of the proposed method and the traditional method for ninelevel phase quantization.

maximum amplitude after quantization is 1.14 and the minimum amplitude is 0.86 with optimized $m=1 / 7$, so the extinction ratio can be calculated as $E X T=10 \log \left(1.14^{2} / 0.86^{2}\right)=$ $2.45 d B$. The phase difference between two adjacent steps is $\pi / 4$ on phase-phase transfer curve. It can be seen that there is a positive correlation between the extinction ratio and steepness of the quantizer phase response. The higher value of EXT means the better quantization effect, which is more preferred when the quantizer is applied to the all-optical ADC because the target quantized phases can be easier selected when the amplitude differences at the target phases are larger.

\section{B. Nine-level Phase Quantization}

In order to explain the theory as shown in Eq. (2) in detail when $M$ is odd, we take the nine-level all-optical phase quantization as an example $(M=9)$. The 4 th phase harmonics conjugate and the 5th phase harmonic are needed according to the Eq. (2), and the output signal can be described as follows:

$$
\left|A_{\text {out }}\right| e^{i \varphi_{\text {out }}}=e^{-i 4 \varphi_{\text {in }}}+m_{2} e^{i 5 \varphi_{\text {in }}}
$$




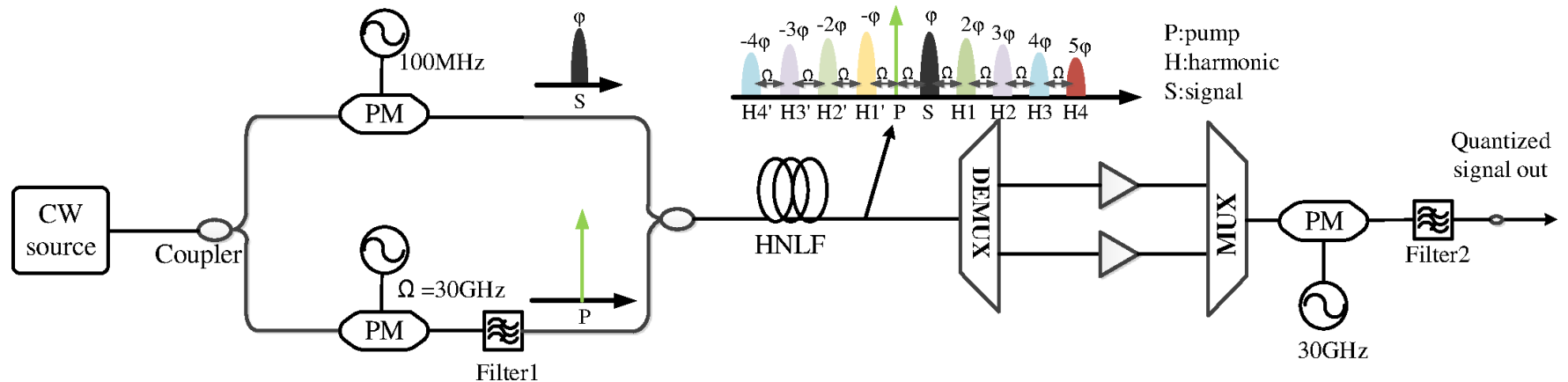

Fig. 3. Principle scheme of the eight- and nine-level all-optical phase quantization using the proposed method.

Where, $m_{2}$ is the amplitude ratio between the 5th phase harmonic and the 4 th phase harmonic conjugate. The constellation diagram and the phase-phase transfer characteristic of the ninelevel phase quantization using the traditional method as Eq. (1) are illustrated in Fig. 2 (a) and (b) with $\varphi_{\text {in }}$ ranging from $-\pi$ to $\pi$ and optimized $m=1 / 8$, respectively. The constellation diagram and the phase-phase transfer characteristic using the proposed method with $\varphi_{i n}$ ranging from $-\pi$ to $\pi$ and optimized $m_{2}=0.83$ are respectively shown in Fig. 2 (c) and (d). The target quantized phases of $\varphi=0, \pm 2 \pi / 9, \pm 4 \pi / 9$, $\pm 6 \pi / 9, \pm 8 \pi / 9$ are squeezed and amplified, and the quadrature phases are attenuated. Thus, the nine-level all-optical phase quantization is achieved.

The phase-phase transfer characteristics with different $m_{2}$ where the target phase around the zero are shown in Fig. 2 (e). The nine-level quantization shows the optimal effect when $m_{2}$ is around 0.83. The phase response can be written as Eq. (5) when $M$ is odd. By Eq. (5), we have $\left.\frac{d F}{d \varphi_{i n}}\right|_{\varphi_{i n}=0}=0$ when $m_{2}=[(M-1) / 2] /[(M+1) / 2]$, which means that the phasephase transfer curve will be flat near the zero phase point. Similarly, phase-phase transfer curve will be flat near the zero phase point when $m_{1}=(M / 2-1) /(M / 2+1)$ for $M=4 n$; and when $m_{11}=(M / 2-2) /(M / 2+2)$ for $M=4 n+2$. Similar to the eight-level phase quantization, for the nine-level phase quantization, when amplitude ratios are all the optimum values, the amplitude differences between target phases and the quadrature phases are larger than those by the traditional method, as shown in Fig. 2(f). The extinction ratio of the proposed scheme is $20.64 \mathrm{~dB}$, while for traditional method the extinction ratio is $2.18 \mathrm{~dB}$.

$$
\begin{aligned}
\varphi_{\text {out }} & =F\left(\varphi_{\text {in }}\right) \\
& =\tan ^{-1}\left[\frac{m_{2} \sin \left(\frac{M+1}{2} \varphi_{i n}\right)+\sin \left(\frac{1-M}{2} \varphi_{i n}\right)}{\cos \left(\frac{1-M}{2} \varphi_{i n}\right)+m_{2} \cos \left(\frac{M+1}{2} \varphi_{i n}\right)}\right]
\end{aligned}
$$

\section{Simulation Configuration}

The system simulation is implemented by solving the coupled nonlinear Schrodinger equations (NLSE) with following the approach presented by Marhic [21]. We mainly consider the following nonlinear processes during the signals transmission in HNLFs: four-wave mixing (FWM), self-phase modulation (SPM), cross-phase modulation (XPM). Other nonlinear effects, such as stimulated Brillouin scattering (SBS)

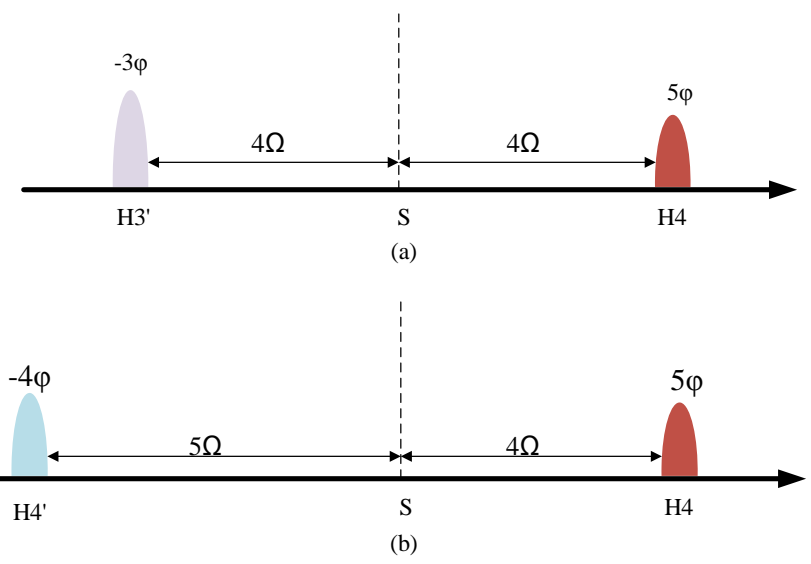

Fig. 4. The frequency configurations of (a) eight-level phase quantization; (b) nine-level phase quantization.

and stimulated Raman scattering (SRS) are not taken into consideration.

The simulation configuration of realizing the eight- and nine-level quantization is shown in Fig. 3. A narrow-line width continuous-wave $(\mathrm{CW})$ source at $1553.6 \mathrm{~nm}(193.1 \mathrm{THz})$ is split into two portions. One portion is modulated by a $100 \mathrm{MHz}$ sinusoidal signal to generate the phase modulated signal with phase ranging from $-\pi$ to $\pi$, and the average power of $10.8 \mathrm{dBm}$. The other portion is sent into a phase modulator (PM) driven by a sinusoidal signal with a frequency of $\Omega=30 \mathrm{GHz}$ to generate an optical frequency comb. Then, the optical wave at $1553.84 \mathrm{~nm}(193.07 \mathrm{THz})$ is selected from the optical frequency comb and amplified as the pump with the average power of $21.0 \mathrm{dBm}$. The pump and the signal are amplified to about $24.4 \mathrm{dBm}$ and sent to the $500 \mathrm{~m}$ HNLF to generate the needed harmonics. The HNLF has a zerodispersion wavelength of $1553.6 \mathrm{~nm}(193.1 \mathrm{THz})$, nonlinear coefficient of $13.1 W^{-1} \mathrm{~km}^{-1}$ and dispersion slope of 0.08 $\mathrm{psnm}^{-2} \mathrm{~km}^{-1}$. For eight-level phase quantization, the $3 \mathrm{rd}-$ order phase harmonic conjugate at $192.98 \mathrm{THz}$ and the 5thorder phase harmonic at $193.22 \mathrm{THz}$ are filtered out, and the powers are amplified to $-0.1 \mathrm{dBm}$ and $-3.0 \mathrm{dBm}$ respectively. Subsequently the two harmonics are launched into a phase modulator (PM) driven by a sinusoidal signal with a frequency of $\Omega$. The up-converted 3rd-order harmonic conjugate and the 


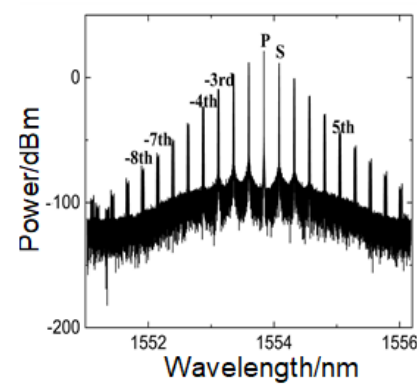

(a)

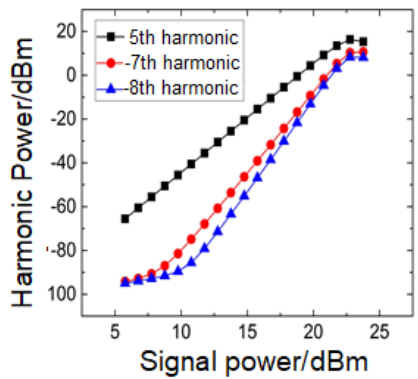

(b)
Fig. 5. (a) The optical spectrum after FWM when the signal and pump power are set as $13.8 \mathrm{dBm}$ and $24.0 \mathrm{dBm}$, respectively; (b) The optical powers of harmonics with the signal power increasing from $5.8 \mathrm{dBm}$ to $23.8 \mathrm{dBm}$.

down-converted 5th-order harmonic waves are converted to the same frequency of $193.1 \mathrm{THz}$ and interfere with each other. After the frequency is up- and down-shifted by $4 \Omega$, as is shown in Fig. 4(a), the power of 3rd-order phase harmonic conjugate is $-16.5 \mathrm{dBm}$, and the power of 5 th-order phase harmonic changes to $-19.4 \mathrm{dBm}$. Finally, the optical quantized signal is generated at the same frequency as the original signal.

In practical, due to the time-varying phase of signals and pumps transmitted in optical fibers, it is essential to apply a phase-locked loop (PLL) in order to maintain a stable phase relationship between the pump and the signal [22]. A radio frequency (RF) signal and Mach-Zehnder modulator (MZM) can also be utilized to generate optical frequency combs which are used as the phase-locking waves in the schemes [19]. Besides, a method named phase locking free can also implement phase locking, which locally generates a suitable set of phase-locked carriers and transfers the phase modulation of an incoming data signal to one of those carriers without disturbing the phase relationship between the pumps and the data signal carrier [23]. Some other details have been considered in practical scenarios. In order to make the system work well, the PM controlled by a voltage-controlled oscillator (VCO) can be used as a frequency shifter to make the two harmonics adding coherently at the same frequency precisely. In such a scenario, a small part of the output optical signal is divided to a photo detector (PD) for monitoring the output optical power and provides a feedback control to the VCO. For nine-level phase quantization, the 4th-order phase harmonic conjugate and 5th-order phase harmonic are selected, and the powers are amplified to $7.2 \mathrm{dBm}$ and $-3.5 \mathrm{dBm}$ respectively. The rest of the steps are same as those in the eight-level phase quantization. As is shown in Fig. 4(b), the 4th-order phase harmonic conjugate is up-shifted by $5 \Omega$ and its power changes to $-18.5 \mathrm{dBm}$ after the frequency shifting. Besides, the power of 5 th-order phase harmonic changes to $-19.9 \mathrm{dBm}$ after the frequency are down-shifted by $4 \Omega$.

\section{RESUlTS AND Discussion}

\section{A. The Results of Eight- and Nine-Level Phase Quantization}

The optical spectrum of FWM in the HNLF with pump and signal power conditions set as those used in the simulation is shown in Figure. 5(a). The power of the 3rd-order harmonic

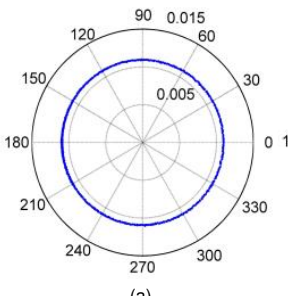

(a)

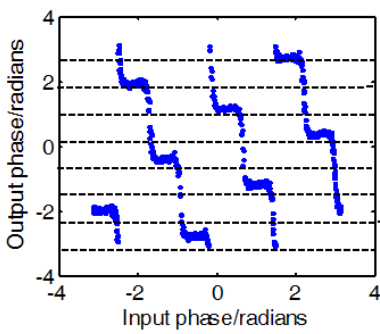

(d)

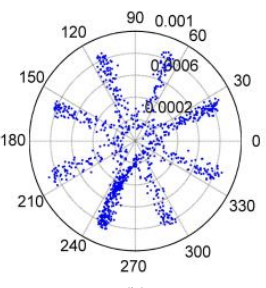

(b)

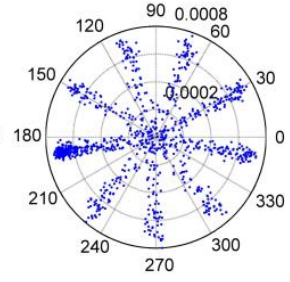

(c)

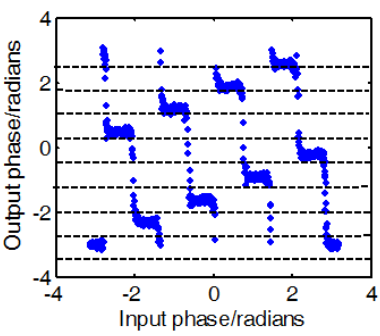

(e)
Fig. 6. The constellation diagrams of (a) input signal, (b) eight-level phase quantization, and (c) nine-level phase quantization with the optimized amplitude ratio of their respective harmonics; The phase-phase characteristics of (d) eight-level phase quantization and (e) nine-level phase quantization.

conjugate, 5th-order harmonic and 7th-order harmonic conjugate are $-5.1 \mathrm{dBm},-25.5 \mathrm{dBm}$ and $-53.6 \mathrm{dBm}$, respectively. In addition, the variation of optical powers of the harmonics are simulated with the power of signal increasing from $5.8 \mathrm{dBm}$ to $23.8 \mathrm{dBm}$ when the pump power is $24.0 \mathrm{dBm}$. The results are illustrated in Fig. 5(b). It is shown that when the signal power changes from $5.8 \mathrm{dBm}$ to $23.8 \mathrm{dBm}$, the power of the 5 th-order harmonic is always higher than that of the 7 th-order harmonic conjugate or the 8th-order harmonic conjugate. In other words, for both eight- and nine-level phase quantization governed by Eq. (3) and Eq. (4) respectively, the power of the 5 th-order harmonic needed in the proposed scheme is higher than that of the 7 th-order or 8th-order harmonic conjugates required by the conventional scheme as Eq. (1).

The constellation diagrams of the input signal, eight-level phase quantization and nine-level phase quantization with the optimal amplitude ratio of their respective harmonics are illustrated in Fig. 6(a), (b) and (c), respectively. The phase-phase characteristics of eight-level phase quantization and nine-level phase quantization are shown in Fig. 6(d) and (e), respectively. The phase distributions of the quantized signal concentrate on their target phases as seen from the constellation diagrams. Simulation results validate that the proposed method of quantization makes the phase-phase transfer characteristic to be the step-like curve. Although the curve is not identical to the strictly monotonically increasing step-like curve, the output phase is discrete and unique for each input phase, and most output phases concentrate on the target phases as quantized. The eight- and nine-level all-optical phase quantization are achieved by using low-order harmonics.

\section{B. The Regeneration of $8 P S K$ signal}

The proposed scheme can be used for all-optical regeneration of the multi-level phase shift keying signal. By replacing 


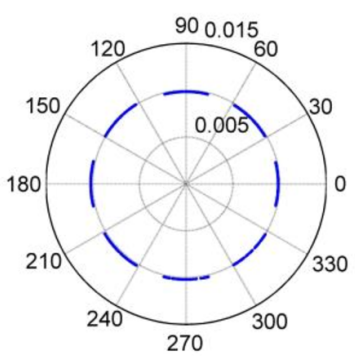

(a)

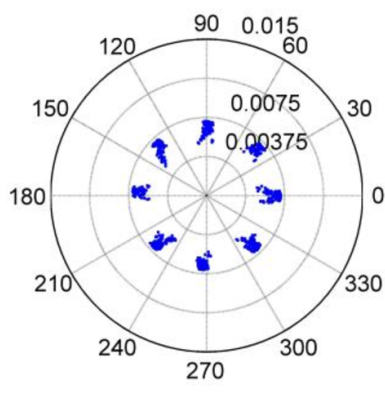

(c)

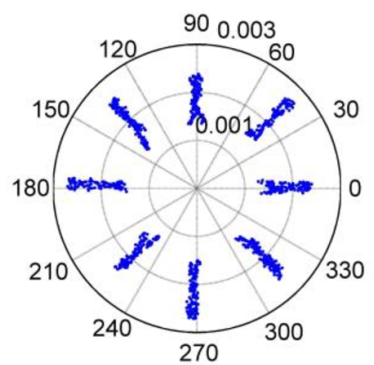

(b)

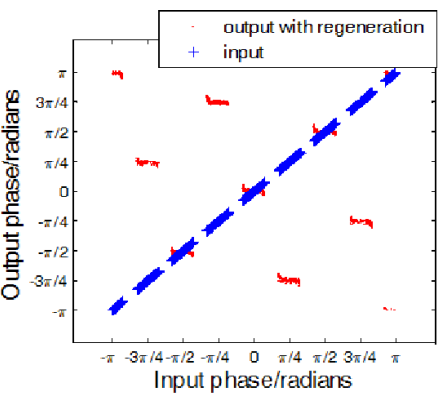

(d)
Fig. 7. Constellation diagrams of (a) input 8PSK signal with 27-degree phase noise, and (b) the regenerated signal by the eight-level phase quantization scheme, and (c) the output signal after the following phase-preserving amplitude regeneration. (d) The phase-phase transfer characteristics at the input (plus sign) and at the output after amplitude regeneration (dot mark).

the all-phase optical signal by an 8PSK signal with a symbol rate of 10GBaud carrying with phase noise, the eight-level phase quantization scheme can be used to the regeneration of the noise contaminated 8PSK signal. In order to get the better effect of phase regeneration, the conjugate 3rd- and 5th-order harmonics are generated by cascading HNLFs in series. The original signal $(193.1 \mathrm{THz}, 9.8 \mathrm{dBm})$ and the pump $(193.07 \mathrm{THz}$, $22.0 \mathrm{dBm}$ ) generated by the method as shown in the simulation configuration are sent into the first HNLF with a length of $500 \mathrm{~m}$, nonlinear coefficient of $13.1 \mathrm{~W}^{-1} \mathrm{~km}^{-1}$ and dispersion slope of $0.08 \mathrm{psnm}^{-2} \mathrm{~km}^{-1}$. After the FWM process, the phase conjugate of the signal is generated. Then, the signal and its phase conjugate $(193.04 \mathrm{THz}, 9.6 \mathrm{dBm})$ are selected and injected to the second HNLF with length of $500 \mathrm{~m}$, nonlinear coefficient of $13.1 \mathrm{~W}^{-1} \mathrm{~km}^{-1}$ and dispersion slope of 0.08 psnm $^{-2} \mathrm{~km}^{-1}$ to generate the conjugate 3rd-order harmonic. Subsequently, the conjugate 3rd-order harmonic $(192.98 \mathrm{THz}$, $-12.5 \mathrm{dBm}$ ) and the original signal are amplified to $16.8 \mathrm{dBm}$ and injected to the next HNLF with length of $300 \mathrm{~m}$, nonlinear coefficient of $17.5 \mathrm{~W}^{-1} \mathrm{~km}^{-1}$ and dispersion slope of 0.08 psnm $^{-2} \mathrm{~km}^{-1}$ to generate the 5th-order harmonic. Finally, the conjugate 3rd- and the 5th-order harmonics are amplified to $1.1 \mathrm{dBm}$ and $-5.4 \mathrm{dBm}$ respectively and are then sent to the PM driven by a sinusoidal signal with frequency of $4 \Omega$ to add the harmonics coherently at the frequency of the original signal.

Fig. 7(a) and (b) show the constellation diagrams of the 8PSK signal with 27-degree phase noise before and after regeneration, respectively. At the output of the regeneration, it is obvious that the phase noises on the 8PSK signal are squeezed effectively. The phase standard deviation is reduced from 9.6 to 2.1 degrees. However, phase noise is converted

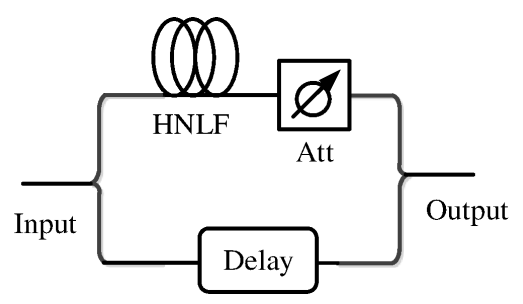

Fig. 8. The phase-preserving amplitude regeneration device.

to amplitude noise via the cosine transfer function after the phase regeneration. In order to avoid amplitude noise being converted to nonlinear phase noise again during the subsequent transmission due to the Gordon-Mollenauer effect which is one of the major limiting factors for phase-encoded signal [24], it is necessary to perform the amplitude regeneration whilst phase preservation in the follow-up system [25]. The phasepreserving amplitude regeneration configuration is depicted in Fig. 8. It can be seen from Fig. 8 that the device is only composed of HNLF, attenuator, delay and coupler, where HNLF has a length of $1000 \mathrm{~m}$, nonlinear coefficient of 13.1 $W^{-1} \mathrm{~km}^{-1}$, and dispersion slope of $0.08 \mathrm{psnm}^{-2} \mathrm{~km}^{-1}$, attenuator has an attenuation of $2 \mathrm{~dB}$ to adjust the power ratio between the signals in two branches. The input signal in lower branch needs to undergo a delay before adding to the signal in upper branch to compensate the transmission phase shift brought by HNLF. Amplitude regeneration is achieved by coherent addition of the SPM-based phase-modulated state and the unaffected one. Two or more stages can be cascaded in order to achieve a better amplitude noise compression effect. Fig. 7(c) shows the constellation diagram of the amplified output signal after the phase-preserving amplitude regeneration. At the output of amplitude regeneration, the phase standard deviation is 2.9 degrees and the error vector magnitude (EVM) which is calculated utilizing the method in [26] is reduced from $16.5 \%$ at the input to $8.8 \%$ at the output. The phasephase transfer characteristics of the input signal and the final output are also shown in Fig. 7(d). This clearly demonstrates the fact that the all-optical phase regeneration is achieved. The input phase $\{-\pi,-3 \pi / 4,-\pi / 2,-\pi / 4,0, \pi / 4, \pi / 2$, $3 \pi / 4\}$ correspond to output phases $\{-\pi, \pi / 4,-\pi / 2,3 \pi / 4,0$, $-3 \pi / 4, \pi / 2,-\pi / 4\}$, respectively. In other words, the output regenerated target phases $\varphi_{\text {out }}$ are corresponding to $-3 \varphi_{\text {in }}$. ( $\varphi_{i n^{\prime}}$ is the original input phase without noises). Following this rule, we can decode the output phase states corresponding to the input phase states at the receiver.

In reality, there are no signals carrying only phase noise, so the simulations of the 8PSK signal carrying both amplitude noise satisfying Gaussian distribution and phase noise evenly distributed over 0 to $2 \pi$ are performed in the same simulation system to better reveal the regeneration performance of the proposed scheme. The input 8PSK signal with a symbol rate of 10GBaud has an OSNR value of $20 \mathrm{~dB}$, amplitude standard deviation of 0.0013 , phase standard deviation of 6.7 degrees and its power is set to be $9.8 \mathrm{dBm}$, as is shown in Fig. 9 (a). The constellation diagram of 8PSK signal after phase regeneration is illustrated in Fig. 9(b), which shows that the 


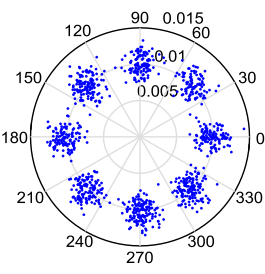

(a)

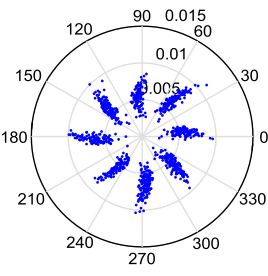

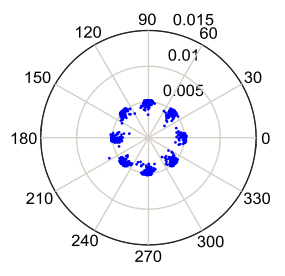

(c)
Fig. 9. Constellation diagrams of the (a) input 8PSK signal carrying Gaussian white noise with OSNR of 20dB; (b) the regenerated 8PSK signal by the eight-level phase quantization scheme; (c) the output 8PSK signal after the following phase-preserving amplitude regeneration.

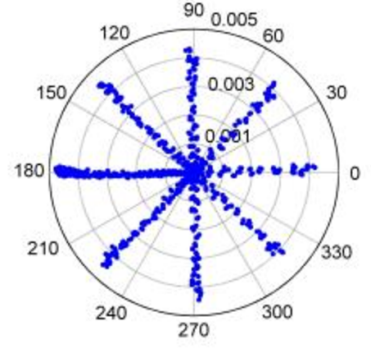

(a)

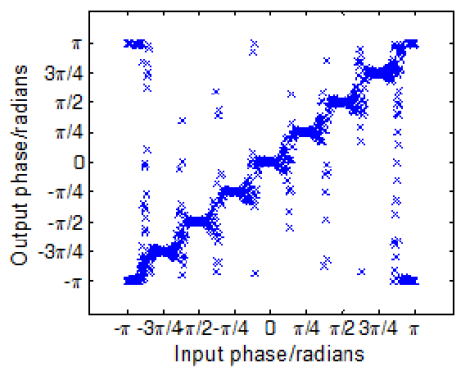

(b)
Fig. 10. (a) Constellation diagram and (b) phase-phase transfer characteristics of the final output signal of the eight-level phase quantization by the improved two-stage cascading method.

phase distribution of the 8PSK signals after phase regeneration concentrates on their target phases very well. At the output of phase regeneration, the phase standard deviation is reduced to 4.3 degrees. Then it is necessary to carry out phase-preserving amplitude regeneration in the subsequent system. Fig .9(c) shows the constellation of 8PSK signal after amplitude and phase regeneration. It is evident that there is a large extent of compression on phase and amplitude compared to the contaminated 8PSK signal as illustrated in Fig .9(a). The amplitude standard deviation of the signal in Fig .9(c) is reduced to $3 \times 10^{-4}$, the phase standard deviation is reduced to 4.1 degrees and the EVM is reduced from $16.9 \%$ to $10.7 \%$. It can be seen from the above simulation results that the proposed scheme has a good compression effect on the phase noise, and the subsequent amplitude regeneration can also significantly reduce the amplitude noise of the signal with phases preserved.

In order to make the phase-phase transfer function strictly monotonically increasing step-like curve and to make the proposed all-optical phase quantization scheme most suitable for phase regeneration, one feasible method is to cascade two stages of phase quantization with the same configuration as shown in Fig. 3. As an example, simulations are implemented for the eight-level phase quantization by using the two-stage cascading method with each one configured as in Fig. 3. The constellation diagrams and phase-phase transfer characteristics of the final output signal are shown in Fig. 10(a) and (b) respectively. The simulation results indicate that the twostage cascading method makes the quantized phases more concentrated on the target phases comparing with results by the one-stage quantization method shown in Fig. 6(b) and (d). More importantly, the two-stage cascading method achieves the monotonically increasing step-like phase-phase transfer characteristics. For phase regeneration, this monotonically and step-like phase-phase transfer characteristics makes the output regenerated target phases $\varphi_{\text {out }}$ corresponding to $\varphi_{\text {in }}$, instead of $-3 \varphi_{i n}$. In Fig. 10(b), there are some spiky points at the junction of two steps, however, for all-optical phase regeneration they can be ignored. The reason is that the phase noise of the input signal for regeneration is much smaller than that of an all-phase signal, which results that the phases of the output signal only fall on the level of the staircase.

\section{CONCLUSION}

In this study, an all-optical multi-level phase quantization method with high-efficiency was proposed and verified by coherently adding two low-order harmonics based on phase sensitive amplification. By the new method, the $M$-level alloptical phase quantization can be achieved by combining two harmonics with the order of the $(M / 2-1) t h$ conjugate and the $(M / 2+1)$ th for $M=4 n$, or the $(M / 2-2) t h$ conjugate and the $(M / 2+2) t h$ for $M=4 n+2$, or the $[(M-1) / 2]$ th conjugate and the $[(M+1) / 2] t h$ for $M=2 n+1$. Simulations were implemented to validate the effectiveness of the proposed method by applying it to the eight- and ninelevel all-optical phase quantization. The scheme of eightlevel phase quantization was also applied to the all-optical phase regeneration of an 8PSK signal, and the phase standard deviation of the regenerated signal was reduced from 9.6 to 2.9 degrees after the phase-preserving amplitude regeneration. A feasible improvement method with two stages cascading was also proposed and validated to achieve the monotonically increasing step-like phase-phase transfer characteristic to make the scheme widely applied to the all-optical phase regeneration in a better way. The proposed method makes it possible to avoid the difficulty of high-order harmonics generating, thus provides a new way and makes it more effectual and easier for multi-level phase quantization or MPSK regeneration to meet the ever increasing demand for the bandwidth in fiber optic communication.

\section{REFERENCES}

[1] J. Kakande, R. Slavík, F. Parmigiani, A. Bogris, D. Syvridis, L. Grünernielsen, R. Phelan, P. Petropoulos, and D. J. Richardson, "Multilevel quantization of optical phase in a novel coherent parametric mixer architecture," Nature Photonics, vol. 5, no. 12, pp. 748-752, 2011.

[2] Y. Ji, J. Zhang, Y. Zhao, X. Yu, J. Zhang, and X. Chen, "Prospects and research issues in multi-dimensional all optical networks," Sci. China Inf. Sci, vol. 59, no. 10, p. 101301, 2016.

[3] T. Konishi, K. Takahashi, H. Matsui, T. Satoh, and K. Itoh, "Five-bit parallel operation of optical quantization and coding for photonic analogto-digital conversion," Optics Express, vol. 19, no. 17, pp. 16106-14, 2011.

[4] K. Itoh, T. Konishi, T. Nagashima, and T. Satoh, "Power-saving approach toward 7-bit optical quantization for photonic analog-to-digital conversion," in Lasers and Electro-Optics Pacific Rim, 2013, p. TuO1.2.

[5] Z. Kang, X. Zhang, J. Yuan, X. Sang, Q. Wu, G. Farrell, and C. Yu, "Resolution-enhanced all-optical analog-to-digital converter employing cascade optical quantization operation," Optics Express, vol. 22, no. 18, pp. 21441-21 453, 2014.

[6] T. Kodama, K. Morita, G. Cincotti, and K. Kitayama, "A low-power photonic quantization approach using ofdm subcarrier spectral shifts," Optics Express, vol. 22, no. 23, pp. 28719-30, 2014. 
[7] R. Slavík, F. Parmigiani, J. Kakande, C. Lundström, M. Sjödin, P. A. Andrekson, R. Weerasuriya, S. Sygletos, A. D. Ellis, and L. Grünernielsen, "All-optical phase and amplitude regenerator for next-generation telecommunications systems," Nature Photonics, vol. 4, no. 10, pp. 690695,2010

[8] K. R. H. Bottrill, L. Jones, F. Parmigiani, and D. J. Richardson, "Fwm-based, idler-free phase quantiser with flexible operating power," in Optical Fiber Communications Conference. Optical Society of America, 2015, p. OW1I.3.

[9] N.-K. Kjøller, K. M. Røge, P. Guan, H. C. H. Mulvad, M. Galili, and L. K. Oxenløwe, "A novel phase-locking-free phase sensitive amplifierbased regenerator," Journal of Lightwave Technology, vol. 34, no. 2, pp. 643-652, 2016.

[10] N. K. Kjøller, F. D. Ros, K. M. Røge, M. Galili, and L. K. Oxenløwe, "Qpsk regeneration without active phase-locking," in Conference on Lasers and Electro-Optics. Optical Society of America, 2016, p. JTh2A.119.

[11] P. Guan, F. D. Ros, M. Lillieholm, H. Hu, K. M. Roge, M. Galili, T. Morioka, and L. Oxenlowe, "16 channel wdm regeneration in a single phase-sensitive amplifier through optical fourier transformation," in Proc. Eur. Conf. Opt. Commun., 2016, pp. 1-3.

[12] B. Schmauss, B. Stiller, G. Leuchs, and G. Onishchukov, "Phase regeneration of a star-8qam signal in a phase-sensitive amplifier with conjugated pumps," Optics Express, vol. 22, no. 1, pp. 1028-1035, 2014.

[13] H. Wang, F. Li, and Y. Ji, "All-optical simultaneous phase and amplitude regenerator based on a modified mach-zehnder interferometric phase sensitive amplifier," Optical Engineering, vol. 56, no. 2, p. 026101, 2017.

[14] K. R. Bottrill, G. D. Hesketh, F. Parmigiani, P. Horak, D. J. Richardson, and P. Petropoulos, "An optical phase quantiser exhibiting suppressed phase dependent gain variation," in Optical Fiber Communications Conference. Optical Society of America, 2014, p. W3F.7.

[15] A. Bogris, "Optical phase processor with enhanced phase quantization properties for higher order modulation formats relying on phase sensitive amplification and limiting amplifiers," in The European Conference on Lasers and Electro-Optics. Optical Society of America, 2015, p. CI.P.13.

[16] A. Bogris and E. Pikasis, "Enhanced multi-level phase quantizer for the optical processing of m-psk signals," Journal of Lightwave Technology, vol. 34, no. 10, pp. 2571-2577, 2016.

[17] H. Wang, C. He, G. Li, and Y. Ji, "All-optical phase quantization with high accuracy based on a multi-wave interference phase sensitive amplifier," IEEE Photon. J., vol. 9, no. 3, p. 7802508, 2017.

[18] J. Y. Yang, Y. Akasaka, and M. Sekiya, "Optical phase regeneration of multi-level psk using dual-conjugate-pump degenerate phase-sensitive amplification," in Optical Fiber Communications Conference. Optical Society of America, 2012, p. P3.07.

[19] T. Kurosu, M. Gao, K. Solis-Trapala, and S. Namiki, "Phase regeneration of phase encoded signals by hybrid optical phase squeezer," Optics Express, vol. 22, no. 10, p. 12177, 2014.

[20] H. Wang, T. Liu, K. Tong, Y. Ji, and L. Bai, "Analytical solution of amplitude ratio in optical phase quantization based on phase sensitive amplification," Journal of Lightwave Technology, vol. 36, no. 16, pp. 3198-3204, 2018.

[21] M. E. Marhic, Fiber Optical Parametric Amplifiers, Oscillators and Related Devices, 1st ed. New York, NY, USA: Cambridge University Press, 2007.

[22] T. Umeki, M. Asobe, and H. Takenouchi, "In-line phase sensitive amplifier based on ppln waveguides," Opt. Express, vol. 21, no. 10, pp. 12077-12 084, May 2013.

[23] N. K. Kjøller, K. M. Røge, P. Guan, H. C. H. Mulvad, M. Galili, and L. K. Oxenløwe, "A novel phase-locking-free phase sensitive amplifier based regenerator," in Optical Fiber Communication Conference. Optical Society of America, 2015, p. W4C.2.

[24] K. Croussore, I. Kim, C. Kim, Y. Han, and G. Li, "Phase-and-amplitude regeneration of differential phase-shift keyed signals using a phasesensitive amplifier," Opt. Express, vol. 14, no. 6, pp. 2085-2094, Mar 2006.

[25] Z. Bakhtiari and A. A. Sawchuk, "All-optical tunable multilevel amplitude regeneration based on coherent wave mixing using a polarizer," Optics Express, vol. 23, no. 8, pp. 10533-9, 2015.

[26] R. Schmogrow, B. Nebendahl, M. Winter, A. Josten, D. Hillerkuss, S. Koenig, J. Meyer, M. Dreschmann, M. Huebner, and C. Koos, "Error vector magnitude as a performance measure for advanced modulation formats," IEEE Photonics Technology Letters, vol. 24, no. 1, pp. 61-63, 2011. 